1997-05-01

\title{
Secondary dark rings of internal conical refraction
}

Karl F. Warnick

warnick@byu.edu

David V. Arnold

Follow this and additional works at: https://scholarsarchive.byu.edu/facpub

Part of the Electrical and Computer Engineering Commons

\section{Original Publication Citation}

Warnick, Karl F., and David V. Arnold."Secondary dark rings of internal conical refraction."

Physical Review E. A 55.5 (1997): 692-96

\section{BYU ScholarsArchive Citation}

Warnick, Karl F. and Arnold, David V., "Secondary dark rings of internal conical refraction" (1997). Faculty Publications. 668.

https://scholarsarchive.byu.edu/facpub/668

This Peer-Reviewed Article is brought to you for free and open access by BYU ScholarsArchive. It has been accepted for inclusion in Faculty Publications by an authorized administrator of BYU ScholarsArchive. For more information, please contact ellen_amatangelo@byu.edu. 


\title{
Secondary dark rings of internal conical refraction
}

\author{
Karl F. Warnick and David V. Arnold \\ Department of Electrical and Computer Engineering, 459 Clyde Building, Brigham Young University, Provo, Utah, 84602
}

(Received 11 June 1996; revised manuscript received 4 November 1996)

\begin{abstract}
This paper treats the phenomenon of internal conical refraction, in which a narrow beam propagating along an optical axis of a biaxial anisotropic medium spreads into a hollow cone. An expression is obtained for the intensity distribution produced by conical refraction that predicts additional fringes concentric to the wellknown Poggendorf dark ring for certain propagation distances and beam widths. [S1063-651X(97)04005-1]
\end{abstract}

PACS number(s): 42.25.Bs, 42.25.Lc, 78.20.Ci, 78.20.Fm

\section{INTRODUCTION}

A narrow beam propagating along one of the optical axes of a biaxial medium spreads into a hollow cone. This phenomenon, internal conical refraction, was predicted by Hamilton in 1832 and observed shortly thereafter by Lloyd. A dark ring in the circular intensity pattern produced by conical refraction was observed by Poggendorf in 1839 and later explained by Voigt. (These historical references and an elementary treatment of conical refraction are found in Born and Wolf [1].) Voigt's explanation of the Poggendorf dark ring was made more precise by Portigal and Burstein [2]. Lalor [3] and Juretschke [4] also analyzed internal conical refraction. Schell and Bloembergen [5] further refined the analysis of Portigal and Burstein, achieving a result accurate to second order in an angle away from the optical axis. Despite the improved accuracy, Schell and Bloembergen employed numerical integration in order to obtain some of the results given in the paper. Other theoretical treatments include that of Uhlmann [6], who proves the existence of the dark ring but does not examine the structure of the intensity pattern in detail.

Previous theoretical treatments amount to a twodimensional stationary phase evaluation of an inverse Fourier transform integral for the refracted field intensity. Our treatment employs the wave-vector representation of the dyadic Green function for the electric field given by Lax and Nelson [7]. A conical expansion for the wave surface near an optical axis given by Moskvin, Romanov, and Val'Kov [8] yields a paraxial approximation for the Green function. The refracted fields can then be obtained by finding the inverse Fourier transform of the product of the Green function and the spectral representation of a Gaussian beam. We treat asymptotically an integration in azimuthal angle about the optical axis, and the remaining transverse integration can be evaluated analytically. The resulting simple characterization of the intensity pattern in terms of special functions is one of the primary contributions of this paper to the theory of internal conical refraction. In order to demonstrate the validity of our approach, we have also performed numerical integrations for the field intensity at certain parameter values.

Our results agree with the theoretical and experimental results of Schell and Bloembergen [5] for a $1 \mathrm{~cm}$ Aragonite sample, a $34 \mu \mathrm{m}$ beam waist, and a wavelength of 0.6328 $\mu \mathrm{m}$. For a $10 \mathrm{~cm}$ sample length, however, their theoretical results are qualitatively similar to the $1 \mathrm{~cm}$ pattern, whereas our treatment predicts secondary dark rings or fringes in the interior of the cone of refraction. We specify the parameter ranges for which this secondary oscillatory behavior of the intensity pattern should appear, and demonstrate that even allowing for large variation of the parameters the effect persists. These secondary dark rings have apparently not been predicted by past theoretical treatments, nor have experimental results been given for parameter values lying within this oscillatory regime.

Measurements by Schell and Bloembergen [9] indicate the appearance of qualitatively similar secondary rings for conical refraction by an optically active medium. Oscillatory behavior of the intensity pattern has been predicted for conical refraction in gyrotropic media $[10,11]$, but the field has an Airy function dependence and is identically zero for certain distances from the cone of refraction. This behavior is qualitatively different from that reported here for biaxial media. Other related work includes that of Naida [12], who considers conical refraction in an inhomogeneous, weakly biaxial medium. Belskii [13] obtains transmission coefficients for a thin biaxial plate along the optical axes, and Belskii and Khapalyuk [14] discuss the change in astigmatism of a Gaussian beam propagating along an optical axis. Khatkevich [15] shows that a conically refracted beam is not confined to a particular generator of the cone, and plane wave solutions near the optical axis are discussed by Alexandroff [16]. References [10,11,17] also investigate the application of conical refraction in gyrotropic media to beam focusing. A recent experimental measurement for conical refraction in potassium titanyl phosphate is found in Ref. [18].

\section{PROPAGATION ALONG AN OPTICAL AXIS}

To evaluate the electric field intensity due to internal conical refraction, we employ the Fourier representation of the tensor Green function for a biaxial, nonmagnetic medium with a real, symmetric permittivity tensor. The field can then be obtained from the inverse Fourier transform of the product of the Green function and the spectral representation of a narrow, Gaussian beam.

The tensor Green function for a biaxial, nonmagnetic material is

$$
G(\mathbf{k}, \omega)=\left[k^{2}(1-\hat{\mathbf{n}} \hat{\mathbf{n}})-\omega^{2} \mu \overline{\bar{\epsilon}}\right]^{-1},
$$

where $\omega$ is the time frequency, $\mathbf{k}=k \hat{\mathbf{n}}$ is the wave vector, $\mu$ is the permeability of the medium, and $\overline{\bar{\epsilon}}$ is the real, symmet- 


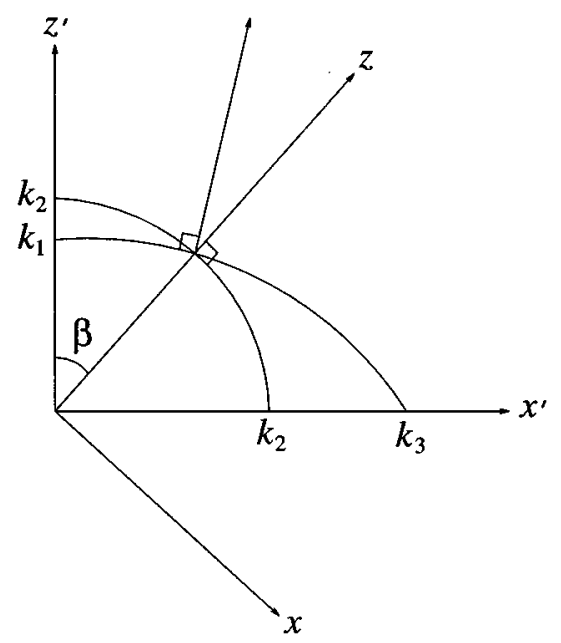

FIG. 1. Geometry of conical refraction. The $z$ direction is an optical axis. Normals to the wave surface at the singular point generate the cone of refraction.

ric permittivity tensor of the medium. The Green function can be conveniently represented by a spectral decomposition [7]

$$
G=\sum_{j=1}^{3} \frac{\mathbf{v}_{j} \mathbf{v}_{j}}{\omega^{2} \mu\left(k^{2} / k_{j}^{2}-1\right)},
$$

where the polarization vectors $\mathbf{v}_{j}$ satisfy $\left[k^{2}(1-\hat{\mathbf{n}} \hat{\mathbf{n}})\right.$ $\left.-\omega^{2} \mu \overline{\bar{\epsilon}}\right] \mathbf{v}_{j}=0$ and are normalized, such that

$$
\mathbf{v}_{j} \overline{\bar{\epsilon}} \mathbf{v}_{j}=1 \text {. }
$$

The $k_{j}$ are the corresponding solutions to the Fresnel equation $\operatorname{det}\left[k^{2}(1-\hat{\mathbf{n}} \hat{\mathbf{n}})-\omega^{2} \mu \overline{\bar{\epsilon}}\right]=0$. One of the $k_{j}$ is infinite; the associated term of Eq. (2) is the nonpropagating part of $G$.

The wave surface defined by the Fresnel equation consists of two sheets, corresponding to the two nonzero solutions for each wave-vector direction $\hat{\mathbf{n}}$. The external and internal sheets of the wave surface meet at singular points along the optical axes or binormals. Let $\left(x^{\prime}, y^{\prime}, z^{\prime}\right)$ be the principle coordinate system of the permittivity tensor. If the eigenvalues are ordered so that $\epsilon_{1}<\epsilon_{2}<\epsilon_{3}$, then the optical axes lie in the $x^{\prime}-z^{\prime}$ plane, at angles

$$
\tan \beta= \pm\left(\frac{\epsilon_{3}\left(\epsilon_{2}-\epsilon_{1}\right)}{\epsilon_{1}\left(\epsilon_{3}-\epsilon_{2}\right)}\right)^{1 / 2}
$$

from the $z^{\prime}$ axis. Near these directions, the wave surface forms a cone. Let $x, y$, and $z$ be the rotated coordinates

$$
\begin{gathered}
x=x^{\prime} \cos \beta-z^{\prime} \sin \beta, \\
y=y^{\prime}, \\
z=x^{\prime} \sin \beta+z^{\prime} \cos \beta,
\end{gathered}
$$

so that the $z$ axis lies in the direction of one of the optical axes. The geometry is depicted in Fig. 1.

In cylindrical coordinates associated with the rotated coordinate system, the wave surface has an expansion about $k_{\rho}=0$ of the form $k_{z}=T_{j}$, where [8]

$$
\begin{gathered}
T_{j}=k_{2}+A\left[\cos \phi+(-1)^{j+1}\right] k_{\rho}-B_{j}(\phi) k_{\rho}^{2}, \\
B_{j}(\phi)=B\left[1+(-1)^{j} D \cos \phi\right]\left[1-(-1)^{j} E \cos \phi\right],
\end{gathered}
$$

and $k_{2}=\omega \sqrt{\epsilon_{2} \mu}$. The $j=1$ term corresponds to the external part of the wave surface and $j=2$ to the inner part. The constants are

$$
\begin{gathered}
A=\frac{1}{2}\left(\frac{\left(\epsilon_{3}-\epsilon_{2}\right)\left(\epsilon_{2}-\epsilon_{1}\right)}{\epsilon_{1} \epsilon_{3}}\right)^{1 / 2}, \\
B=\frac{\left(\epsilon_{3}+\epsilon_{2}\right)\left(\epsilon_{2}+\epsilon_{1}\right)}{8 \epsilon_{1} \epsilon_{3} k_{2}}, \\
D=\frac{\epsilon_{3}-\epsilon_{2}}{\epsilon_{3}+\epsilon_{2}}, \\
E=\frac{\epsilon_{2}-\epsilon_{1}}{\epsilon_{2}+\epsilon_{1}} .
\end{gathered}
$$

The apex angle of the cone of refraction is $2 A$.

Neglecting the nonpropagating term, the tensor Green function for small $k_{\rho}$ is

$$
G=\sum_{j=1}^{2} \frac{\epsilon_{2} \mathbf{v}_{j} \mathbf{v}_{j}}{k_{z}^{2}-T_{j}^{2}},
$$

where the $\mathbf{v}_{j}$ are in the principle coordinate system. The polarization vectors $\mathbf{v}_{j}$ can be found using the geometric relationship between the electric displacement vector $\mathbf{D}$ and the wave and ray vectors. The vectors $\mathbf{D}_{j}$ corresponding to the $\mathbf{v}_{j}$ either lie in the plane containing the wave vector and the ray vector or are perpendicular to it. For $\mathbf{k}$ along the $z$ axis and ray vectors lying on the cone of refraction,

$$
\mathbf{D}_{1}=\hat{\mathbf{x}} \cos \frac{\phi}{2}+\hat{\mathbf{y}} \sin \frac{\phi}{2}
$$

and $\mathbf{D}_{2}=\mathbf{D}_{1}(\phi+\pi)$. The $\mathbf{v}_{j}$ are proportional to $\overline{\bar{\epsilon}}^{-1} \mathbf{D}_{j}$, so that

$$
\begin{aligned}
\mathbf{v}_{1}= & N\left[\hat{\mathbf{x}}^{\prime} \epsilon_{1}^{-1} \cos \beta \cos (\phi / 2)+\hat{\mathbf{y}}^{\prime} \epsilon_{2}^{-1} \sin (\phi / 2)\right. \\
& \left.-\hat{\mathbf{z}}^{\prime} \epsilon_{3}^{-1} \sin \beta \cos (\phi / 2)\right],
\end{aligned}
$$

with the normalization $N=\left[\epsilon_{1}^{-1} \cos ^{2} \beta \cos ^{2}(\phi / 2)\right.$ $\left.+\epsilon_{2}^{-1} \sin ^{2}(\phi / 2)+\epsilon_{3}^{-1} \sin ^{2} \beta \cos ^{2}(\phi / 2)\right]^{-1 / 2}$. The eigenvector $\mathbf{v}_{2}$ is $\mathbf{v}_{1}(\phi+\pi)$.

The refracted electric field due to a Gaussian beam of waist size $w_{0}$ focused at one face of the biaxial medium is then

$$
\mathbf{E}=\frac{i \omega \mu}{8 \pi^{3}} \int d \mathbf{k} e^{i \mathbf{k} \cdot \mathbf{r}} G(\mathbf{k}) \xi_{0}\left(k_{\rho}\right) \hat{\mathbf{p}}
$$

where $\xi_{0}\left(k_{\rho}\right)=\left(2 E_{0} / \eta_{2}\right) \pi w_{0}^{2} e^{-w_{0}^{2} k_{\rho}^{2} / 4}, \eta_{2}=\sqrt{\mu / \epsilon_{2}}$, and $\hat{\mathbf{p}}$ specifies the polarization of the beam in the principle coordinate system. Integrating $k_{z}$ by a contour closing in the upper half plane yields 


$$
\begin{aligned}
\mathbf{E}= & -\frac{k_{2} E_{0}}{8 \pi^{2} \omega} \int k_{\rho} d k_{\rho} d \phi e^{i k_{\rho}(x \cos \phi+y \sin \phi)} \xi_{0}\left(k_{\rho}\right) \\
& \times \sum_{j} \mathbf{v}_{j}\left(\mathbf{v}_{j} \cdot \hat{\mathbf{p}}\right) e^{i T_{j} z},
\end{aligned}
$$

for $z>0$. The leading order phase as a function of $\phi$ for both the external and internal terms is

$$
g(\phi)=(x+A z) \cos \phi+y \sin \phi .
$$

The phase is stationary at two angles; for each of the two terms one of the stationary points is nonphysical. The causal stationary points are

$$
\cos \phi_{j}=(-1)^{j} \frac{(x+A z)}{\sqrt{(x+A z)^{2}+y^{2}}},
$$

where the signs are chosen by noting that $\phi_{j}$ specifies the angle of the point on the external or internal sheet of the wave surface at which the surface normal is in the direction of the ray to the point $(x, y, z)$. Integrating Eq. (11) by the method of stationary phase gives

$$
\begin{aligned}
\mathbf{E}= & -\frac{k_{2} w_{0}^{2} E_{0}}{4 \pi \omega \eta_{2}} e^{i k_{2} z} \sum_{j} \sigma_{j} \mathbf{v}_{j}\left(\mathbf{v}_{j} \cdot \hat{\mathbf{p}}\right) \\
& \times \int k_{\rho} d k_{\rho}\left(\frac{2 \pi}{k_{\rho}\left|g_{j}^{\prime \prime}\right|}\right)^{1 / 2} e^{i k_{\rho} b_{j}-k_{\rho}^{2} a_{j}},
\end{aligned}
$$

where

$$
\begin{gathered}
\sigma_{j}=\exp \left[i(-1)^{j+1} \frac{\pi}{4}\right], \\
g_{j}\left(\phi_{j}\right)=(-1)^{j+1} \sqrt{(x+A z)^{2}+y^{2}}=-g_{j}^{\prime \prime}\left(\phi_{j}\right), \\
a_{j}=\frac{w_{0}^{2}}{4}+i B_{j}\left(\phi_{j}\right) z, \\
b_{j}=(-1)^{j+1}\left[A z-\sqrt{(x+A z)^{2}+y^{2}}\right],
\end{gathered}
$$

and the $\mathbf{v}_{J}$ are evaluated at the stationary point $\phi_{j}$. The large parameter is $k_{\rho} \sqrt{(x+A z)^{2}+y^{2}}$, so that the stationary phase condition becomes invalid at $k_{\rho}=0$. For typical parameter values, however, the resulting error is not significant since the integrand vanishes as $k_{\rho} \rightarrow 0$.

The remaining $k_{\rho}$ integration yields

$$
\begin{aligned}
\mathbf{E}= & -\frac{\boldsymbol{\epsilon}_{2} w_{0}^{2} e^{i k_{2} z} E_{0}}{4 \sqrt{\pi}\left[(x+A z)^{2}+y^{2}\right]^{1 / 4} a_{1}^{3 / 4}} \mathbf{v}_{1}\left(\phi_{1}\right) \\
& \times\left[\mathbf{v}_{1}\left(\phi_{1}\right) \cdot \hat{\mathbf{p}}\right] F\left(a_{1}, b_{1}\right),
\end{aligned}
$$

where

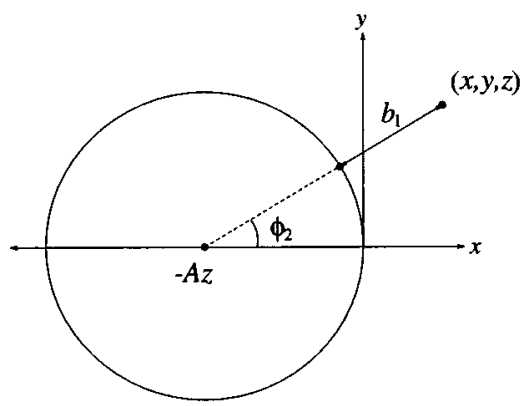

FIG. 2. A circular cross section of the cone of refraction. $b_{1}$ is the distance from $(x, y, z)$ to the cone in the $x-y$ plane.

$$
\begin{aligned}
F(a, b)= & e^{-b^{2} /(4 a)}\left[\Gamma(1 / 2) \Gamma(5 / 4) L_{1 / 4}^{-1 / 2}\left(\frac{b^{2}}{4 a}\right)\right. \\
& \left.-\frac{b}{\sqrt{a}} \Gamma(3 / 2) \Gamma(3 / 4) L_{-1 / 4}^{1 / 2}\left(\frac{b^{2}}{4 a}\right)\right]
\end{aligned}
$$

and $L_{q}^{p}(x)$ is the associated Laguerre function. The internal and external terms combine since they differ only by the sign of $b_{j}$ and the phase $\sigma_{j}$. For large $z, a_{1}$ is approximately $i B_{1} z$, so that the asymptotic dependence of $E$ is $z^{-5 / 4}$, which matches the result reported by Moskvin, Romanov, and Val'skov [8] for the field due to a point source in directions lying on the cone of internal conic refraction. For fixed $z$, the leading behavior of Eq. (19) at large distances from the cone of refraction in the $x-y$ plane is the Gaussian term $\exp \left[-b_{1}^{2} /\left(4 a_{1}\right)\right]$, where $b_{1}$ is the distance from the circular section of the cone with radius $A z$ and center at $(-A z, 0, z)$ as shown in Fig. 2. The polarization term $\mathbf{v}_{1}\left(\phi_{1}\right)\left[\mathbf{v}_{1}\left(\phi_{1}\right)\right.$ $\cdot \hat{\mathbf{p}}]$ modulates the intensity pattern as a function of angle around the cone in the $x-y$ plane, as exhibited by Fig. 3 of Ref. [5].

In order to verify our approach, the $k_{\rho}$ integration of Eq. (11) can be evaluated in terms of associated Laguerre functions and the $\phi$ integration performed numerically. Results obtained in this manner for Aragonite $\left(n_{x}=1.530, n_{y}\right.$ $\left.=1.680, n_{z}=1.685[5]\right), z=10 \mathrm{~cm}$, beam waist $34 \mu \mathrm{m}$, vacuum wavelength $0.6328 \mu \mathrm{m}$, and incident polarization in the $x$ direction differ from the approximate expression (19) by less than $2 \%$ over most of the intensity pattern, as shown in Fig. 3.

The oscillatory behavior of $F(a, b)$ includes the wellknown Poggendorf dark ring, but for certain values of the beam waist size, propagation distance, and permittivities of the biaxial medium, additional fringes appear on the inside of the cone, as shown by the plot of $|F(1+i q, b)|$ in Fig. 4. There are two conditions which must be met in order for the secondary oscillatory behavior of the field intensity pattern to appear. First, $a$ must be such that $F$ is oscillatory as $b$ varies. Second, the radius $A z$ of the cone of refraction must be greater than the distance of the first secondary fringe from the cone of refraction. The coefficients $D$ and $E$ are typically much less than unity, so that $B_{j} \simeq B$, and we need only consider the $y=0$ section of the intensity pattern. The parameters $a_{1}$ and $b_{1}$ of Eq. (19) can be rescaled so that $a_{1}=1$ $+i 4 B z / w_{0}^{2}$ and $b_{1}=-2 x / w_{0}$. As can be verified by exam- 


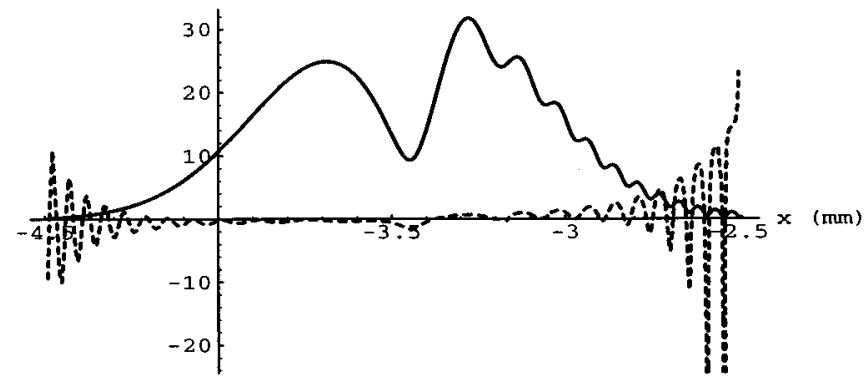

FIG. 3. Magnitude of $10^{3} E / E_{0}$ for Aragonite, $z=10 \mathrm{~cm}$, beam waist $34 \mu \mathrm{m}$, and wavelength $0.6328 \mu \mathrm{m}$ as given by numerical integration of Eq. (11). On the same scale the percentage error of Eq. (19) with respect to the numerical integration is shown as a dashed line. Incident polarization is in the $x$ direction. The cone of refraction intersects the $x$ axis at $x=-3.5 \mathrm{~mm}$.

ining the behavior of $F(1+i q, b)$, the first of the above conditions then yields

$$
0.33<\frac{B z}{w_{0}^{2}}<3.8
$$

for an additional dark ring of at least $10 \%$ variation. The second condition is satisfied if

$$
\frac{A z}{w_{0}}>2.7 \frac{B z}{w_{0}^{2}}+3.0
$$

These ranges are sufficiently large that for reasonable experimental values and parameter variations the oscillatory regime should easily be observed. Secondary dark rings should appear, for example, in the intensity pattern for an Aragonite crystal of length $1 \mathrm{~cm}$, a wavelength of $0.6328 \mu \mathrm{m}$, and a beam waist size of $18 \mu \mathrm{m}$, as shown in Fig. 5. For these values, $A z / w_{0}=9.7$ and $B z / w_{0}^{2}=1.0$, so that both conditions (21) and (22) are satisfied even with large error in the beam waist size, sample length, or permittivities of the medium. The experimental arrangement described by Schell and

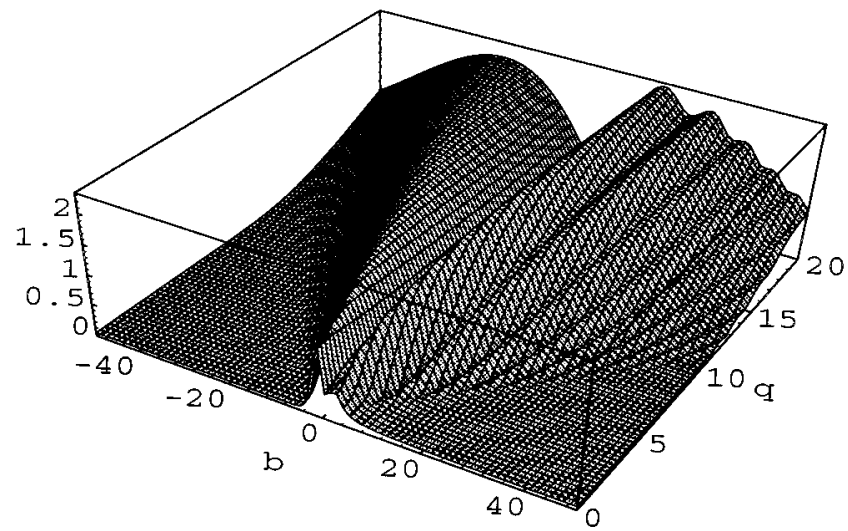

FIG. 4. Magnitude of $F(1+i q, b)$. The local minimum along the $x$ axis produces the dark ring in the intensity pattern of conical refraction. $b>0$ corresponds to the interior of the cone and $b<0$ to the exterior of the cone.

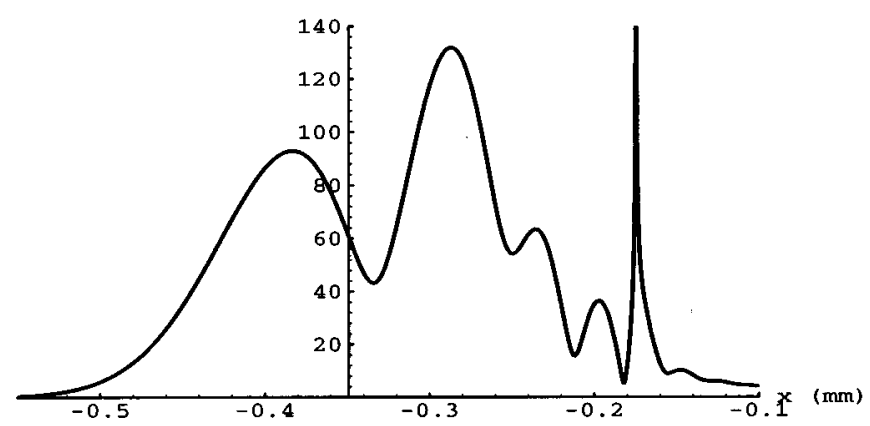

FIG. 5. Magnitude of $10^{3} E / E_{0}$ for Aragonite, $z=1 \mathrm{~cm}$, $w_{0}=18 \mu \mathrm{m}$, and $\lambda=0.6328 \mu \mathrm{m}$. The singularity of Eq. (19) at the center of the cone of refraction appears at $x=-0.175 \mathrm{~mm}$. Incident polarization is in the $x$ direction.

Bloembergen [5] would allow sufficient control of the parameters to remain well within the oscillatory regime of the intensity pattern.

For a $10 \mathrm{~cm}$ crystal length and a beam waist of $34 \mu \mathrm{m}$, our theory also predicts fringing in the intensity pattern (Fig. 6). This does not match the numerical results of Schell and Bloembergen (Fig. 7c of Ref. [5]). Although the shift of the dark ring's minimum towards the interior of the cone and the larger amplitude of the inner peak agree qualitatively, the intensity pattern obtained by Schell and Bloembergen exhibits no additional fringing.

\section{CONCLUSION}

The intensity pattern due to internal conical refraction of a narrow beam by a biaxial medium has a more complicated structure than previously known. Our treatment predicts additional dark rings on the interior of the cone for a range of values of the anisotropy of the medium, beam waist size, and propagation distance. We have given constraints on these parameters for the oscillatory regime of the intensity pattern, and demonstrated that these constraints are physically reasonable and sufficiently large for experimental verification of the effect. Apparently there do not exist, in the literature, measurements of the intensity pattern for beam and material parameters for which secondary dark rings would be expected to appear, and so it seems desirable to further explore

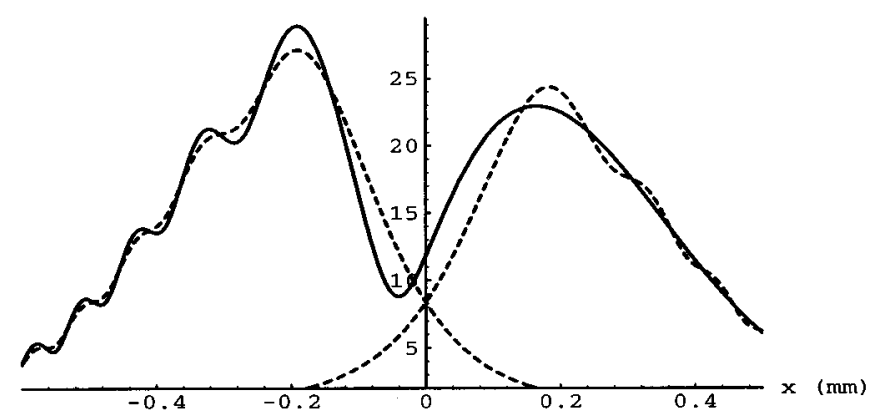

FIG. 6. Magnitude of $10^{3} E / E_{0}$ for Aragonite, $z=10 \mathrm{~cm}, w_{0}$ $=34 \mu \mathrm{m}$, and $\lambda=0.6328 \mu \mathrm{m}$. Dashed lines are magnitudes of the internal and external contributions taken separately and the solid line is total intensity as given by Eq. (19). Incident polarization is in the $y$ direction. 
conical refraction experimentally. Our result also contributes to the theory of internal conical refraction by characterizing the intensity pattern in terms of special functions which make clear the leading behavior of the refracted fields. An extension of this work would be to determine the relationship between the secondary fringes predicted by our theory and those that appear in the intensity pattern for an optically active medium, as reported in Ref. [9].

\section{ACKNOWLEDGMENTS}

K.F.W. would like to thank the National Science Foundation for financial support.
[1] M. Born and E. Wolf, Principles of Optics (Pergamon, Oxford, 1980).

[2] D. L. Portigal and E. Burstein, J. Opt. Soc. Am. 59, 1567 (1969).

[3] E. Lalor, J. Math. Phys. 13, 449 (1972).

[4] H. Juretschke, Crystal Physics: Macroscopic Physics of Anisotropic Solids (Benjamin, New York, 1974).

[5] A. J. Schell and N. Bloembergen, J. Opt. Soc. Am. 68, 1093 (1978).

[6] G. A. Uhlmann, Comm. Pure Appl. Math. 25, 69 (1982).

[7] M. Lax and D. F. Nelson, Phys. Rev. B 4, 3694 (1971).

[8] D. N. Moskvin, V. P. Romanov, and A. Y. Val'kov, Phys. Rev. E 48, 1436 (1993).

[9] A. J. Schell and N. Bloembergen, J. Opt. Soc. Am. 68, 1098 (1978)

[10] Y. A. Brodskii, I. G. Kondratev, and M. A. Miller, Izv. Vyssh. Uchebn. Zaved. Radiofiz. 12, 1339 (1969) [Radiophys. Quan- tum Electron. 12, 1047 (1969)].

[11] Y. A. Brodskii, I. G. Kondratev, and M. A. Miller, Izv. Vyssh. Uchebn. Zaved. Radiofiz. 15, 592 (1972) [Radiophys. Quantum Electron. 15, 447 (1972)].

[12] O. N. Naida, Zh. Eksp. Teor. Fiz. 77, 471 (1979) [Sov. Phys. JETP 50, 239 (1979)].

[13] A. M. Belskii, Opt. Spektrosk. 55, 776 (1983) [Opt. Spectrosc. USSR 55, 465 (1983)].

[14] A. M. Belskii and A. P. Khapalyuk, Opt. Spektrosk. 44, 540 (1938) [Opt. Spectrosc. USSR 44, 312 (1978)].

[15] A. G. Khatkevich, Opt. Spektrosk. 46, 505 (1979) [Opt. Spectrosc. USSR 46, 282 (1979)].

[16] V. N. Alexandroff, J. Opt. 15, 219 (1984).

[17] A. M. Goncharenko et al., Opt. Spektrosk. 78, 872 (1995) [Opt. Spectrosc. USSR 78, 787 (1995)].

[18] J. P. Féve, B. Boulanger, and G. Marnier, Opt. Commun. 105, 243 (1994). 